\title{
Resveratrol attenuates pulmonary embolism associated cardiac injury by suppressing activation of the inflammasome via the MALAT1-miR-22-3p signaling pathway
}

\author{
KAI YANG ${ }^{1}$, WANCHENG LI ${ }^{1}$, WENJUAN DUAN ${ }^{2}$, YI JIANG ${ }^{1}$, NA HUANG ${ }^{1}$, \\ YUNHUI LI ${ }^{1}$, BOCHENG REN ${ }^{1}$ and JIAN SUN ${ }^{1}$ \\ ${ }^{1}$ Respiratory Department, The First Affiliated Hospital of Chengdu Medical College; \\ ${ }^{2}$ Pediatric Respiratory Department, No. 2 Hospital of Chengdu, Chengdu, Sichuan 610500, P.R. China
}

Received November 26, 2017; Accepted April 11, 2019

DOI: $10.3892 /$ ijmm.2019.4358

\begin{abstract}
Resveratrol (RS) has been reported to prevent the development of cardiac injury induced by pulmonary embolism (PE). The present study aimed to explore the potential mechanism of RS involved in cardiac injury induced by PE. A luciferase assay was conducted to detect the effect of RS on promoter efficiency of metastasis associated lung adenocarcinoma transcript 1 (MALAT1), in-silico analysis and luciferase assays were performed to explore the regulatory relationship between MALAT1, microRNA (miR)-22-3p and NLRP3. Reverse transcription PCR, western blot analysis and ELISA were carried out to examine MALAT1, miR-22-3p, NLRP3, ASC, Caspase-1, interleukin (IL)-1 $\beta$ and IL-18 among different animal model groups, including the sham group, PE associated cardiac injury group and PE associated cardiac injury plus RS group. The results revealed that RS downregulated promoter efficiency of MALAT1 and MALAT1 directly targeted miR-22-3p, and luciferase activity of MALAT1 was inhibited by miR-22-3p, and furthermore miR-22-3p inhibited the expression of NLRP 3 by binding to complementary sequences in the 3' untranslated region of NLRP3. MALAT1, NLRP3, ASC, Caspase-1, IL-1 $\beta$ and IL-18 levels were much increased, while miR-22-3p level was much decreased in PE associated cardiac injury group compared with the sham group, while the RS upon the PE associated cardiac injury group slightly reduced the upregulated MALAT1/NLRP3 level and elevated the downregulated miR-22-3p level. In conclusion, it was demonstrated that RS has been demonstrated to prevent the development of cardiac injury induced
\end{abstract}

Correspondence to: Dr Wancheng Li or Dr Bocheng Ren, Respiratory Department, The First Affiliated Hospital of Chengdu Medical College, 278 Baoguangdadao Street, Chengdu, Sichuan 610500, P.R. China

E-mail: lungemboli@126.com

E-mail: bochengren006@yeah.net

Key words: resveratrol, pulmonary embolism associated cardiac injury, inflammasome, NLRP3, MALAT1, miR-22-3p by PE via modulating the expression of MALAT1 and further affect miR-22-3p and NLRP3.

\section{Introduction}

Although significant progress has been made toward the diagnosis and care of acute pulmonary embolism (PE), relevant risk assessment for the disease and proper patient management remains challenging in clinical applications (1). In two previous studies involving large prospective registries, it was demonstrated that, in addition to the manifestations of hemodynamic and clinical instability, acute right ventricular dysfunction is a major prognostic factor in PE patients during their hospitalization $(2,3)$. By monitoring the presence of right ventricular hypokinesis and enlargement in conjunction with echocardiography, patients at a high risk of impending or apparent right ventricular failure can be screened, therefore potentially identifying those patients that may exhibit positive responses to thrombolytic treatments $(4,5)$.

Certain previous studies have demonstrated that $40 \%$ of PE patients with disease severity similar to that in a rat model exhibited persistent right ventricular (RV) dysfunction with restricted cardiopulmonary functions, regardless of their state of comorbidity $(6,7)$. In addition, the monophasic response of neutrophils and a potential biphasic response of monocytes were present throughout the progression and resolution of the cardiac inflammation and during the early stage of scar formation in rats. Therefore, the series of events observed in the rat model suggest the involvement of such inflammatory reactions in post-PE cardiac injuries and indicate that the long-term recovery of heart functions may be enhanced by the management of acute inflammation generated during the disease (8). The activation of inflammasomes, as represented by increased NLRP3 expression, has been shown as a key process during inflammation (9).

As a natural antioxidant, resveratrol is extracted from Polygonum cuspidatum and can decrease the viscosity of blood, inhibit vasodilation and the aggregation of platelets, maintain a normal blood flow, and prevent the onset and progression of cancer (10-12). Ji et al (13) reported that resveratrol could downregulate metastasis associated lung 
adenocarcinoma transcript 1 (MALAT1), therefore decreasing the localization of $\beta$-catenin in the nuclei and attenuating the activity of the Wnt/ $\beta$-catenin signaling pathway, ultimately inhibiting the metastasis of colorectal cancer $(13,14)$.

A total of 10,000s of long noncoding RNAs (lncRNAs), defined as RNA transcripts that have $>200$ nucleotides in length and have no protein coding capabilities, have been identified and a number of these IncRNAs are uniquely expressed in different types of tissues or specific cancers (15). It has been shown that lncRNA genes outnumber the genes responsible for protein-coding, whereas $>90 \%$ of genes in the human genome do not encode peptide products (16-18). It has now been widely recognized that compared to the expression of mRNA, the expression of different lncRNAs is under more strict regulation and therefore is limited to specific cell types (19). As a result, lncRNAs are frequently associated with evolutionarily conserved functions, secondary structures and regions of microhomology, although their overall sequence similarity is low (20-22). It was shown that the expression of MALAT1 was increased in patients suffering from unstable angina. On the other hand, the silencing of MALAT1 expression significantly decreased the expression of CXCR2, a target gene of miR-22-3p, by reversing the function of miR-22-3p, therefore leading to aggravated endothelial injury induced by the oxidization of low-density lipoproteins. The protein kinase B pathway was found to be involved in this entire process (23).

Inflammation has been reported to be an important mechanism underlying the cardiac injury associated with pulmonary embolism and inflammasome with a marker of activated NLRP3 is considered a key signaling pathway of the inflammatory process $(8,24)$. Resveratrol has been reported to treat cardiac or lung injury by suppressing the activation of NLRP3 $(25,26)$. Furthermore, it was noted that administration of resveratrol could downregulate the expression of MALAT1 and MALAT1 functions as a sponge of miR-22-3p $(14,23)$. In this study, the involvement of MALAT1/miR-22-3p/NLRP3 in the therapeutic effect of resveratrol in the treatment of cardiac injury associated with pulmonary embolism was explored.

\section{Materials and methods}

Animals. A total of 24 male Sprague-Dawley rats (age, 50-65 days; weight, 300-375 g) were obtained from the animal center of the First Affiliated Hospital of Chengdu Medical College. The rats were housed at room temperature between $20-25^{\circ} \mathrm{C}$ with a relative humidity between $50-60 \%$. Animals received free access to food and deionized water under a $12 \mathrm{~h}$ light/dark cycle. All experiments were carried out with the agreement of Institutional Animal Care and Use Committee of the First Affiliated Hospital of Chengdu Medical College according to Guide for the Care and Use of Laboratory Animals.

$P E$ model. A total of $3 \mathrm{mg} / \mathrm{kg}$ xylazine and $70 \mathrm{mg} / \mathrm{kg}$ ketamine was utilized to anaesthetize animals and then $2.0 \times 10 \% / 100 \mathrm{~g}$ polystyrene microspheres (Duke Scientific; Thermo Fisher Scientific, Inc.) was utilized to establish PE by injecting into right jugular vein. Animals treated with $0.01 \%$ Tween-20 $(0.16 \mathrm{ml} / 100 \mathrm{~g})$ were used as internal control.
In vivo measurements. There are three groups of rats $(n=8$ per group): Sham groups as the control, animal model of PE group and PE group treated with resveratrol (RS; $30 \mathrm{mg} / \mathrm{kg}$; Santa Cruz Biotechnology, Inc.) solution. A total of $3 \mathrm{mg} / \mathrm{kg}$ xylazine and $70 \mathrm{mg} / \mathrm{kg}$ ketamine was utilized to anaesthetize animals and then placed on warming pads at 6 weeks (a preliminary test was performed to determine the best time point for the treatment group; data not shown). The breathing rate of tested animals was counted and the heart rate and systemic pressures were measured using 2-French micromanometer (Millar Instruments, Inc.), which placed in left carotid artery; RV pressures was detected using 2-French bent Millar micromanometer (Millar Instruments, Inc.) by inserting into jugular vein. Pressure data was acquired using Acknowledge software (ACK100W; Biopac Systems, Inc.). Blood chemistry was analyzed using StatProfile Ultra analyzer (Nova Biomedical) and the blood samples were then stored at $-70^{\circ} \mathrm{C}$ for further analysis.

In vitro $R V$ outflow contractile force measurements. Midline thoracotomy was performed on anaesthetized rats to isolate the heart and then it was stored in ice-cold saline. Krebs-Henseleit bicarbonate buffer was perfused into the aorta for $5 \mathrm{~min}$ at $60 \mathrm{mmHg}$ at $37^{\circ} \mathrm{C}$ in a non-recirculating system. The $\mathrm{RV}$-free walls were isolated and then a strip of muscle was cut longitudinally from the midline of the outflow tract and stored in a tissue bath supplemented with Krebs-Henseleit bicarbonate buffer at $37^{\circ} \mathrm{C}$ with $95 \% \mathrm{O}_{2} / 5 \% \mathrm{CO}_{2}$, and then tied to a fixed point using Refined Myograph System (Kent Scientific Corporation, Torrington, CT, USA). Platinum plate electrodes were used to treat strips at $80 \mathrm{~V}$ and $0.2 \mathrm{~Hz}$ for $10 \mathrm{~ms}$. Acknowledge software (ACK100W; Biopac Systems, Inc.) was used to record data. A total of $0.25 \mathrm{~mm}$ increments in length were used to construct length-tension curves to examine contractile force with optimum length induced maximum active force $\left(\mathrm{mN} / \mathrm{mm}^{2}\right)$. Finally, components were calculated as follow: $\mathrm{mm}^{2}$ cross-section area = to wet $\mathrm{wt} /$ optimum length, then Newton $=$ gm tension $\times 9.807$.

RNA isolation and reverse transcription (RT) PCR. RNeasy total RNA isolation system (Qiagen, Inc.) was used to extract total RNA from HL-1 and AC16 cells and tissue samples. NanoDrop 1000 spectrophotometer (Thermo Fisher Scientific, Inc.) was used to assess RNA purity and concentration. SuperScript ${ }^{\mathrm{TM}}$ III reverse transcriptase (Invitrogen; Thermo Fisher Scientific, Inc.) was utilized to synthesize cDNA of NLRP3. FastStart SYBR Green Master (Roche Diagnostics) was used to carry out quantitative PCR on Mx3005P PCR machine (Agilent Technologies). The reaction was carried out as follows: $50^{\circ} \mathrm{C}$ for $3 \mathrm{~min}, 95^{\circ} \mathrm{C}$ for $12 \mathrm{~min}$, then 40 cycles at $94^{\circ} \mathrm{C}$ for $15 \mathrm{sec}$ and at $60^{\circ} \mathrm{C}$ for $1 \mathrm{~min}$, then $72^{\circ} \mathrm{C}$ for $30 \mathrm{sec}$. Small RNA U6 and GAPDH were served as internal control for MALAT1, miR-22-3P and NLRP3. Comparative $2^{-\Delta \Delta C q}$ method (27) was utilized to calculate relative expressions of MALAT1, miR-22-3P and NLRP3 mRNA, which normalized to expressions of U6 and GAPDH. Each reaction was repeated three times. The primers pairs used were as follows: MALAT1 forward, 5'-CAGACCACCACAGGTTTACAG-3' and reverse, 5'-AGACCATCCCAAAATGCTTCA-3'; miR-22-3P forward, 5'-AAGCTGCCAGTTGAAGAACTGT-3' and reverse, 
5'-CAGTGCGTGTCGTGGAGT-3'; NLRP3 forward, 5'-CTG CAGATGGCAAGCACCCGCTGC-3' and reverse, 5'-CGT TTGGATCCCTACCAAGAAGGCTCAA-3'; U6 forward, 5'-CGCTTCGGCAGCACATATACTA-3' and reverse, 5'-CGC TTCACGAATTTGCGTGTCA-3'; GAPDH forward, 5'- CTT TGTCAAGCTCATTTCCTGG-3' and reverse, 5'-TCTTGC TCAGTGTCCTTGC-3'.

Cell culture and treatment. HL-1 and AC16 cells were obtained from American Type Culture Collection, and incubated with Dulbecco's modified Eagle's medium (Gibco; Thermo Fisher Scientific, Inc.) containing 10\% heat-inactivated fetal bovine serum (Gibco; Thermo Fisher Scientific, Inc.) and antibiotics $(100 \mathrm{mg} / \mathrm{ml}$ streptomycin and $100 \mathrm{U} / \mathrm{ml}$ penicillin; Life Technologies; Thermo Fisher Scientific, Inc.) under a humidified atmosphere with $5 \% \mathrm{CO}_{2}$ at $37^{\circ} \mathrm{C}$ in 24 -well plates for $24 \mathrm{~h}$. When cells reached $50-80 \%$ confluence at a final density of $5 \times 10^{4}$ per well, HL-1 and AC16 cells treated with 1 or $5 \mu \mathrm{m}$ RS were transfected with $30 \mathrm{nM}$ miR-22-3p mimic using Lipofectamine RNA iMAX reagent (Invitrogen; Thermo Fisher Scientific, Inc.) following manufacturer's protocol. RT-qPCR assay was performed to evaluate transfection efficiency. Each test was performed three times.

Cell proliferation assay. MTT assay was performed to assess cell viability of HL-1 and AC16 cells transfected with miR-481 mimics. HL-1 and AC16 cells were cultured in 24-well plates at a final density of $1 \times 10^{4}$ cells/well, and incubated for $48 \mathrm{~h}$, followed by addition with $10 \mu 1$ MTT $(5 \mathrm{mg} / \mathrm{ml})$, and maintained for another $4 \mathrm{~h}$. DMSO was utilized to dissolve the crystals at $37^{\circ} \mathrm{C}$ from $10 \mathrm{~min}$. A spectrophotometer (NanoDrop Technologies) was used to detect optical density values at $490 \mathrm{~nm}$ wavelength. Each experiment was run three times.

Luciferase assay. Using online publicly available algorithms including mirdb.org, miR-22-3p targets were predicted. The full sequence of MALAT1 or NLRP3 3' untranslated region (UTR) containing putative binding site of miR-22-3p was amplified using PCR. The putative binding site of miR-22-3p on MALAT1 or NLRP3 3'UTR was mutated using QuickChange Site-Directed Mutagenesis kit (Stratagene; Agilent Technologies, Inc.) in accordance with the manufacturer's protocol and generated mutant MALAT1/NLRP3 3'UTR. Then wild-type or mutant MALAT1 or NLRP3 3'UTR was sub-cloned into pGL3 vector (Promega Corporation) located on immediately down-stream of luciferase gene. A total of $1 \times 10^{5}$ per well cells were seeded into 48 -well plates, Lipofectiamine $^{\mathrm{TM}} 2000$ (Invitrogen; Thermo Fisher Scientific, Inc.) was utilized to transiently transfect HL-1 and AC16 cells with luciferase construct containing wild-type or mutant MALAT1 or NLRP3 3'UTR and miR-22-3p mimics following manufacturer's protocol. Passive Lysis Buffer (Promega Corporation) was utilized to harvest the cells $36-48 \mathrm{~h}$ post-transfection. Dual luciferase assay system (Promega Corporation) was utilized to detect luciferase activities, which was normalized to Renilla luciferase activity. Each test was performed three times.

Western blot analysis. Ice-cold PBS (Invitrogen; Thermo Fisher Scientific, Inc.) was used to harvest the cells 2 days after transfection and cold-modified radioimmunoprecipitation buffer (Invitrogen; Thermo Fisher Scientific, Inc.) supplemented with $15 \mathrm{mM}$ Tris/HCl pH 7.5, $1 \mathrm{mM}$ EDTA, $25 \mathrm{mM}$ $\mathrm{KCl}, 120 \mathrm{mM} \mathrm{NaCl}, 0.5 \%$ Triton X100, protease inhibitors (Invitrogen; Thermo Fisher Scientific, Inc.) was used to lyse the cells following the manufacturer's protocol. A bicinchoninic acid Protein Assay kit (Bio-Rad Laboratories, Inc.) was used to determine the concentration of the protein based on the manufacturer's protocol. A 4-12\% SDS-PAGE was used to separate total protein ( $35 \mu \mathrm{g}$ per lane) and then transferred on nitrocellulose membranes (Bio-Rad, Laboratories, Inc.). Tris-buffered saline containing $0.1 \%$ Tween-20 (TBST) and $5 \%$ non-fat dry milk (Merck KGaA) was used to block the membrane for $60 \mathrm{~min}$ at room temperature to avoid unspecific binding. Primary rabbit antibodies targeted NLRP3 (cat. no. sc-134306), ASC (cat. no. sc-514414) or Caspase-1 (cat. no. sc-56036; 1;5,000; Santa Cruz Biotechnology, Inc.) or anti- $\beta$-actin (cat. no. A1978; 1;10,000; Sigma-Aldrich; Merck KGaA) were incubated with the membrane at $4^{\circ} \mathrm{C}$ overnight. The secondary diluted horseradish peroxidase (HRP)-conjugated goat anti-rabbit IgG antibody (cat. no. G-21234; 1:10,000; Thermo Fisher Scientific, Inc.) was incubated with the membrane at $4^{\circ} \mathrm{C}$ for $12 \mathrm{~h}$. The protein bands were detected using Bio-Rad ChemiDoc MP Imaging System (Bio-Rad Laboratories, Inc.) with Western Breeze chemilluminescent kit (Invitrogen, Thermo Fisher Scientific, Inc.) following the manufacturer's protocol. Three independent tests were performed.

Apoptosis analysis. Annexin V-fluorescein isothiocyanate (FITC)/PI Apoptosis Detection kit (Sigma-Aldrich; Merck $\mathrm{KGaA}$ ) was used to measure cell apoptosis of HL-1 and AC16 cells using a flow cytometer. In brief, $48 \mathrm{~h}$ after the HL-1 and AC16 cells were transfected with miR-22-3p mimic, the cells were harvested, then washed twice using PBS. 1X binding buffer was used to resuspend cells at a final density of $4 \times 10^{5}$ cells per well. A total of $5 \mu \mathrm{l}$ of PE Annexin $\mathrm{V}$ and $5 \mu \mathrm{l}$ of 7-AAD were used to treat $100 \mu \mathrm{l}$ of cell suspension, and followed by incubation in darkness for 15 min. FACS MELODY ${ }^{\mathrm{TM}}$ (BD Biosciences; Becton, Dickinson and Company) was used to detect cell apoptosis.

ELISA. Levels of IL-1 $\beta$ (cat. no. MLB00C) and IL-18 (cat. no. DY318-05) in serum were tested using ELISA kits (R\&D Systems, Inc.) in accordance with manufacturer's protocol.

Immunohistochemistry. Tissue samples were harvested and cold $4 \%$ paraformaldehyde was used to fix the samples at $4^{\circ} \mathrm{C}$ for $12 \mathrm{~h}$, and samples further embedded in paraffin. Then the samples were cut into 5- $\mu \mathrm{m}$ sections. Hematoxylin and eosin staining were performed to detect expression of NLRP3. 3\% $\mathrm{H}_{2} \mathrm{O}_{2}$ was used to treat sections for 15 min, then $80 \%$ carbinol was incubated with sections for $30 \mathrm{~min}$, then the blocking solution was used to treat sections for another $60 \mathrm{~min}$ at room temperature. Primary rabbit antibodies targeted NLRP3 (cat. no. sc-134306; 1;500; Santa Cruz Biotechnology, Inc.) were incubated with the sections at $4^{\circ} \mathrm{C}$ overnight. PBS was used to wash the sections three times. The secondary diluted HRP-conjugated anti-rabbit IgG antibody (cat. no. G-21234; 
1:2,000; Thermo Fisher Scientific, Inc.) was used to incubate sections at $37^{\circ} \mathrm{C}$ for 2 h. 3,3-diaminobenzidine (DAB) was used to stop the reaction. A Nikon ECLPSE 80i fluorescent microscope (Nikon Corporation, Tokyo, Japan) was used to visualize results. All reactions were carried out in triplicate.

TUNEL. One step TUNEL Apoptosis Asssy kit (Roche $\mathrm{GmbH}$, Mannheim, Germany) was used to detect DNA fragmentation based on the manufacturer's protocol. The 5- $\mu \mathrm{m}$ thick sections were deparaffinized and rehydrated. A total of $10 \mu \mathrm{g} / \mathrm{ml}$ proteinase $\mathrm{K}$ was utilized to treat sections at $37^{\circ} \mathrm{C}$ for $30 \mathrm{~min}$ and $50 \mu \mathrm{l}$ of TUNEL inspection fluid was used to incubate sections at $37^{\circ} \mathrm{C}$ for $60 \mathrm{~min}$, and then washed three times using PBS. DMEM was used as the mounting medium. Nikon ECLIPSE Ti fluorescent microscope (Nikon Corporation) was used to capture the images in three fields of view using $530 \mathrm{~nm}$ wavelength emission and $488 \mathrm{~nm}$ for excitation. Three independent experiments were performed.

Statistical analysis. All results were presented as the mean \pm standard deviation. SPSS19.0 software package (IBM Corporation, Armonk, NY, USA) was used to perform statistical analysis using Student's t test. Additionally, one-way analysis of variance was used to examine the difference between more than two groups and a Scheffe's test was utilized as the post hoc test. $\mathrm{P}<0.05$ was considered to indicate a statistically significant difference.

\section{Results}

Identification the effect of RS on the promoter efficiency of MALAT1. To explore the underlying mechanism of the therapeutic effect of RS in the treatment of PE associated cardiac injury, constructs containing the MALAT1 promoter were obtained and then sub-cloned upstream of the luciferase gene of pcDNA3 (Fig. 1A). Different doses of RS (1 or $5 \mu \mathrm{M})$ were used to treat HL-1 and AC16 cells. Luciferase activity in HL-1 and AC16 cells was significantly dose-dependently downregulated subsequent to transfection with RS compared with the control $(\mathrm{P}<0.05$; Fig. 1B and $\mathrm{C})$, suggesting that RS decreased the promoter efficiency of MALAT1.

MALAT1 directly targets and represses miR-22-3p expression. To validate the successful transfection of miR-22-3p mimics into HL-1 (Fig. 2A) and AC16 (Fig. 2B) cells, relative expression of miR-22-3p was observed, demonstrating significantly upregulated miR-22-3p level in cells transfected with miR-22-3p mimics compared with the control group $(\mathrm{P}<0.05)$. Accordingly, as presented in Fig. 3A and B, a candidate binding site of MALAT1 was determined on miR-22-3p. The potential regulatory relationship between MALAT1 and miR-22-3p was examined using a luciferase assay. miR-22-3p significantly reduced luciferase activity of wild-type MALAT1 but not that of mutant MALAT1 in HL-1 $(\mathrm{P}<0.05$; Fig. $3 \mathrm{C})$ and AC16 $(\mathrm{P}<0.05$; Fig. 3D) cells, suggesting a negative correlation between MALAT1 and miR-22-3p, but this needs to be confirmed.

$N L R P 3$ is the direct downstream of $m i R-22-3 p$. The results from computational analysis demonstrated that a putative binding site of miR-22-3p located within 3'UTR of NLRP3
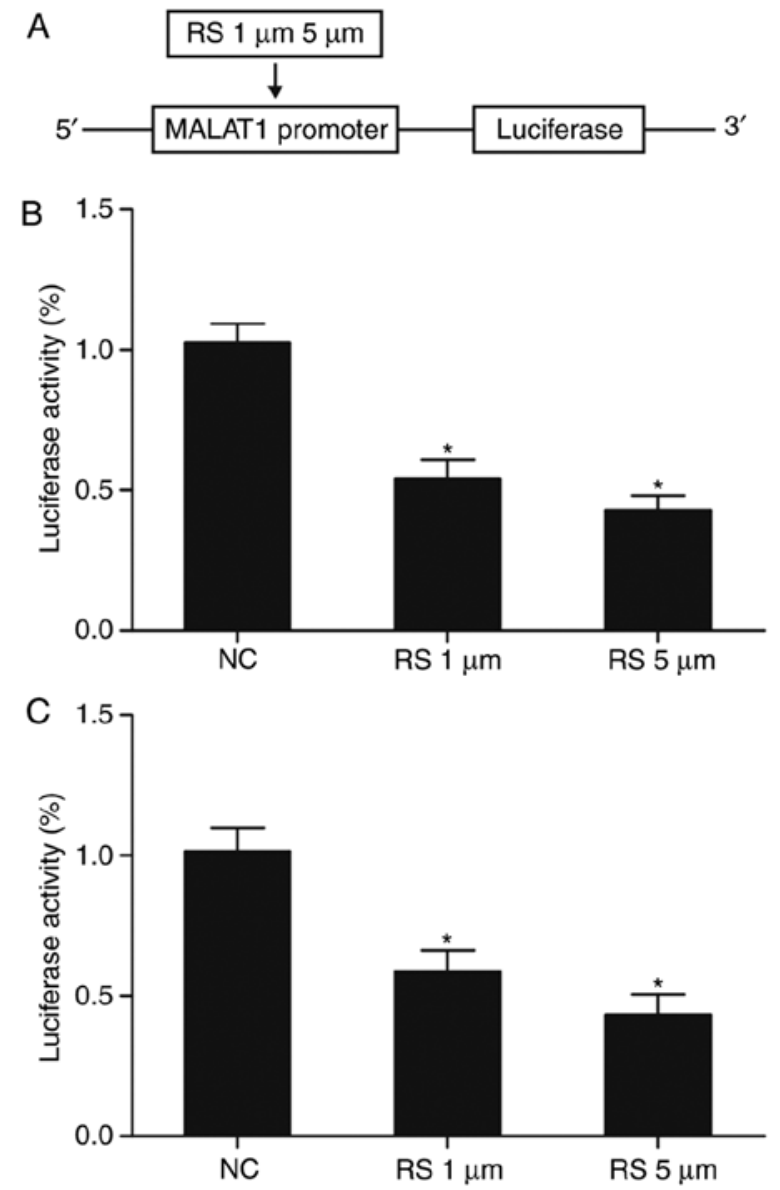

Figure 1. RS increased promoter efficiency of MALAT1. (A) Fragment of MALAT1 promoter was inserted into immediately upstream of luciferase gene. (B) Luciferase activity in HL-1 cells transfected with 1 and $5 \mu \mathrm{m}$ RS compared with the negative controls. ${ }^{*} \mathrm{P}<0.05$ vs. the negative controls. (C) Luciferase activity in AC16 cells transfected with 1 and $5 \mu \mathrm{m}$ RS compared with the negative controls. ${ }^{*} \mathrm{P}<0.05$ vs. the negative controls. RS, resveratrol; MALAT1, metastasis associated lung adenocarcinoma transcript $1 ;$ NC, negative control.

(Fig. 4A). To further verify the regulatory relationship between miR-22-3p and NLRP3, a luciferase reporter plasmid containing the NLRP3 fragment with 3 'UTR or without miR-22-3p binding site was generated, and HL-1 and AC16 cells co-transfected with the above luciferase construct and miR-22-3p mimic or scramble controls. As presented in Fig. 4B and C, the luciferase activity of the NLRP3 fragment with 3'UTR was significantly inhibited by miR-22-3p $(\mathrm{P}<0.05)$ but the luciferase activity of the NLRP3 fragment without the 3'UTR demonstrated no obvious difference compared with the scramble control in HL-1 (Fig. 4C) and AC16 (Fig. 4C) cells. These findings validated that miR-22-3p inhibited the expression of NLRP3 by binding to complementary sequences in the 3'UTR of NLRP3.

MALAT1, miR-22-3p and NLRP3 are differently expressed in different groups. An animal model with PE associated cardiac injury was established and RS was used to treat animal with PE associated cardiac injury. RT-PCR was carried out to detect the expression levels of MALAT1, miR-22-3p and NLRP3 in the sham, PE associated cardiac injury and PE associated cardiac injury plus RS groups. As presented in Fig. 5, the MALAT1 

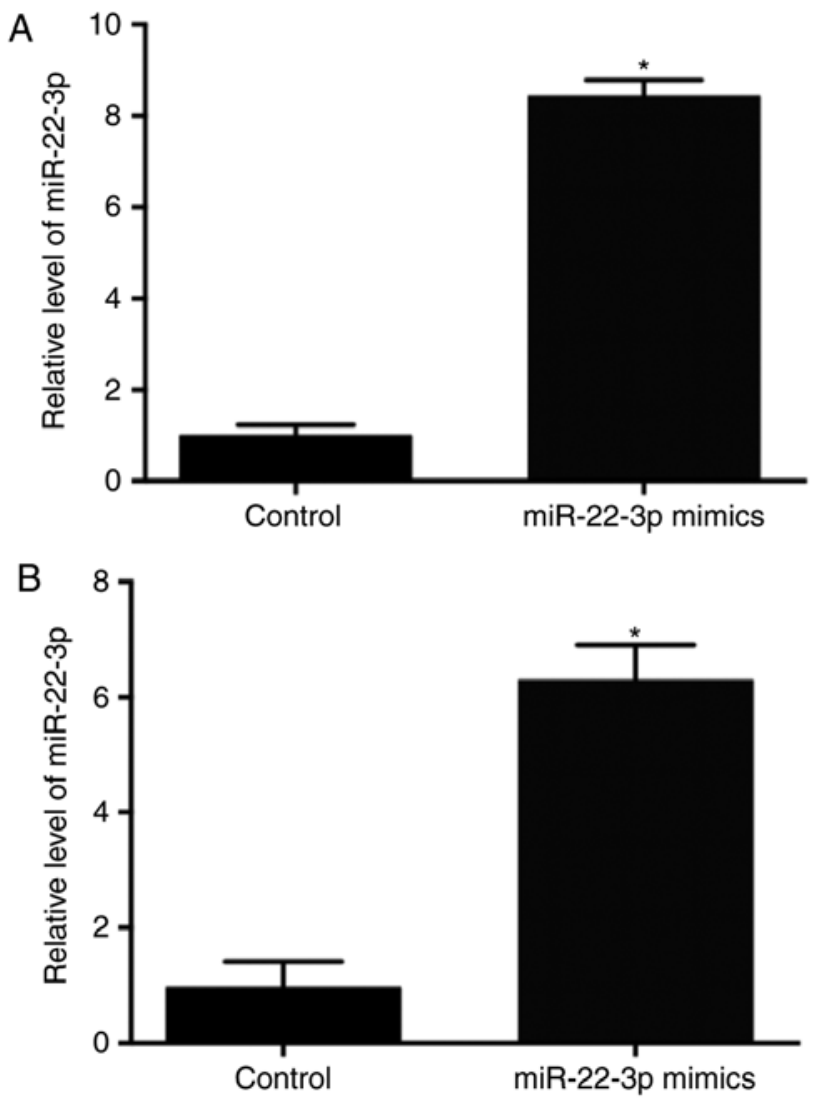

Figure 2. Transfection of miR-22-3p mimics successfully elevated the relative expression of miR-22-3p. (A) Relative expression level of miR-22-3p with or without the transfection of miR-22-3p mimics in HL-1 cells. ${ }^{*} \mathrm{P}<0.05$ vs. the scramble controls. (B) Relative expression level of miR-22-3p with or without the transfection of miR-22-3p mimics in AC16 cells. " $\mathrm{P}<0.05$ vs. the scramble controls. miR, microRNA.

(Fig. 5A) and NLRP3 (Fig. 5C) levels in PE associated cardiac injury group were significantly increased in the sham group $(\mathrm{P}<0.05)$, and the administration of RS reduced the upregulated MALAT1 and NLRP3 levels in the PE associated cardiac injury group, although the level remained higher than the sham group. On the contrary, the miR-22-3p (Fig. 5B) level in PE associated cardiac injury group was significantly decreased compared with the PE associated cardiac injury plus RS group and both groups were much lower than the sham group.

NLRP3, ASC and Caspase-1 is differently expressed in different groups. Western-blot analysis was performed to examine protein levels of NLRP3, ASC and Caspase-1 between the sham, PE associated cardiac injury and PE associated cardiac injury plus RS groups. As presented in Fig. 6, the $\mathrm{PE}$ associated cardiac injury group exhibited significantly increased protein levels of NLRP3 ( $\mathrm{P}<0.05$; Fig. 6A and $\mathrm{B})$, ASC (Fig. 6A and C) and Caspase-1 (Fig. 6A and D) compared with the sham group, while PE associated cardiac injury plus the RS group displayed significantly decreased protein levels of NLRP3, ASC and Caspase-1 than PE associated cardiac injury group $(\mathrm{P}<0.05)$.

Differential levels of inflammatory factors in various groups. ELISA was carried out to determine protein levels of IL-1 $\beta$ and IL-18 among sham, PE associated cardiac injury and PE

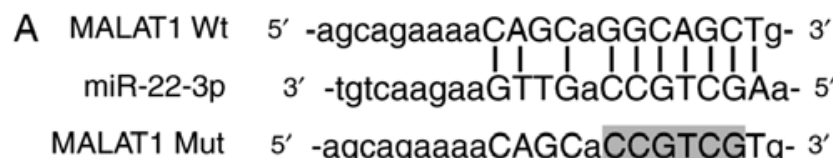

MALAT1 Mut $5{ }^{\prime}$-agcagaaaaCAGCaCCGTCGTg- 3'
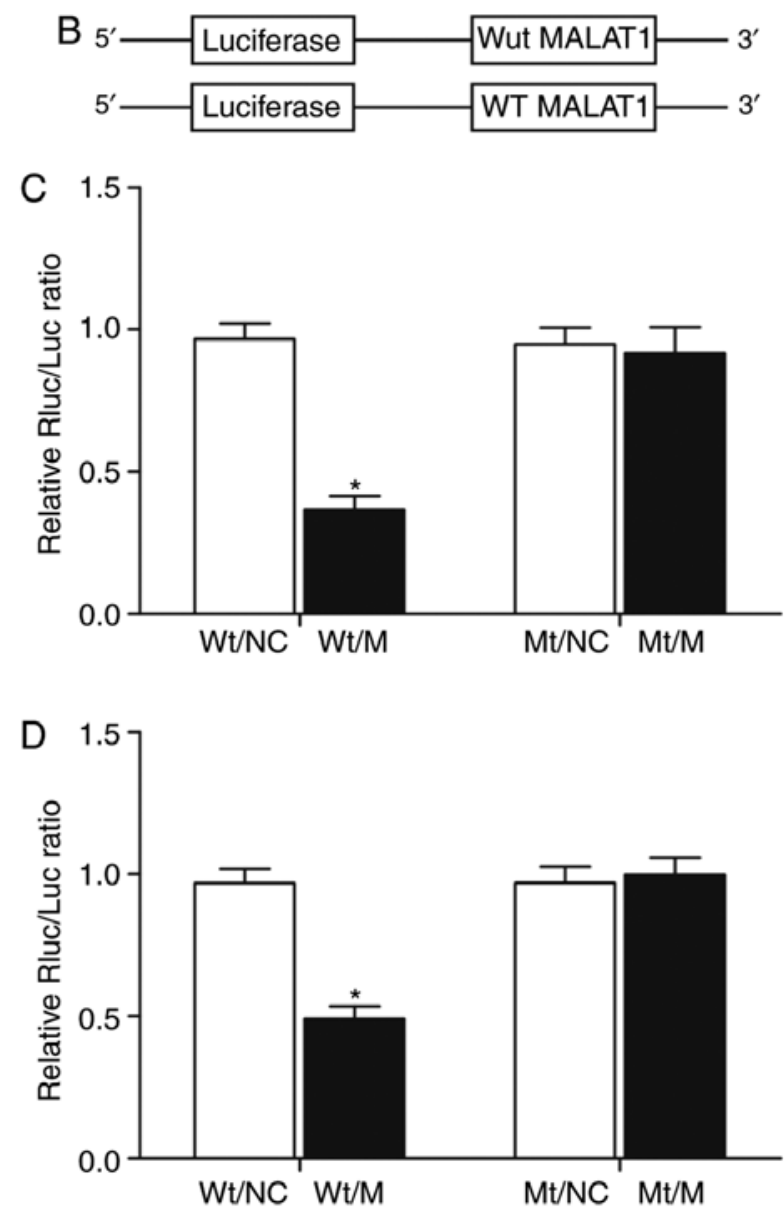

Figure 3. MALAT1 directly targets and represses miR-22-3p expression. (A) Schematic comparison between MALAT1 and the 'seed sequence' in miR-22-3p. (B) Constructs with Wt/M MALAT1 inserted downstream of the luciferase gene. (C) Luciferase activity of HL-1 cells co-transfected with Wt/M MALAT1 and miR-22-3p compared with the scramble controls. ${ }^{*} \mathrm{P}<0.05$ vs. the scramble controls. (D) Luciferase activity of AC16 cells co-transfected with Wt/M MALAT1 and miR-22-p compared with the scramble controls. ${ }^{*} \mathrm{P}<0.05$ vs. the scramble controls. miR, mircoRNA; MALAT1, metastasis associated lung adenocarcinoma transcript $1 ; \mathrm{Wt} / \mathrm{M}$, wild-type/mimics; NC, negative control; Mt/M, mutant type/mimics.

associated cardiac injury plus RS groups. As presented in Fig. 7, IL-1 $\beta$ (Fig. 7A) and IL-18 (Fig. 7B) levels in PE associated cardiac injury group were significantly increased compared with the sham group $(\mathrm{P}<0.05)$, and significantly decreased in $\mathrm{PE}$ associated cardiac injury plus $\mathrm{RS}$ group compared with the $\mathrm{PE}$ associated cardiac injury group $(\mathrm{P}<0.05)$.

Differential NLRP3 protein level in different groups. Immunohistochemistry was performed to examine the protein levels of NLRP3 in the sham, PE associated cardiac injury and $\mathrm{PE}$ associated cardiac injury plus RS groups. As presented in Fig. 8, the PE associated cardiac injury group exhibited higher protein levels of NLRP3 (Fig. 8) compared with the sham group, while PE associated cardiac injury plus RS group displayed a slightly reduced protein levels of NLRP3 (Fig. 8) compared with the PE associated cardiac injury group 

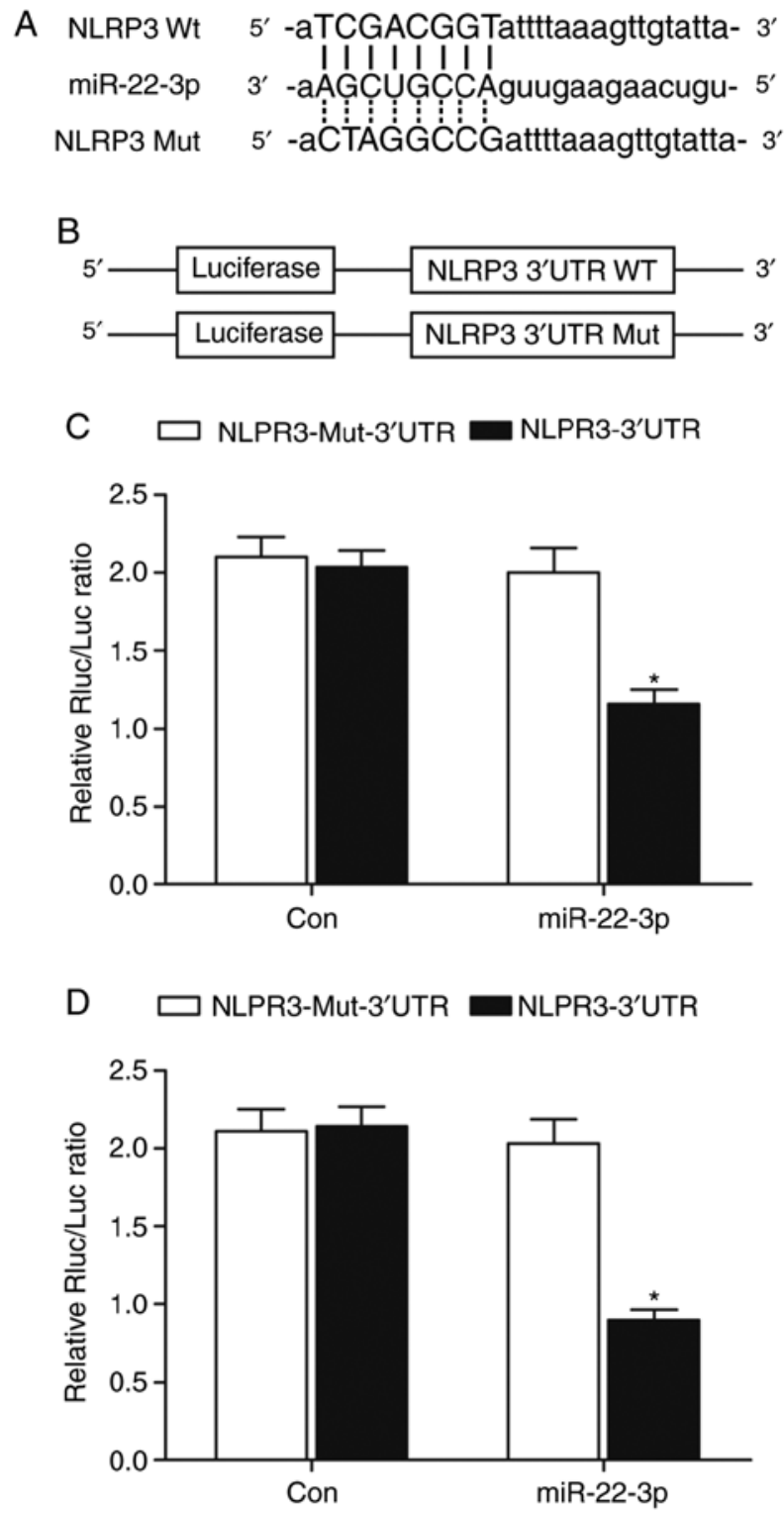

Figure 4. NLRP3 was the direct downstream of miR-22-3p. (A) Schematic comparison between NLRP3 3'UTR and the 'seed sequence' in miR-22-3p. (B) Constructs with wild-type or mutant NLRP3 3'UTR inserted down-stream of the luciferase gene. (C) Luciferase activity of HL-1 cells co-transfected with miR-22-3p/scramble controls and wild-type NLRP3 3'UTR compared with mutant NLRP3 3'UTR. "P<0.05 vs. the mutant NLRP3 3'UTR. (D) Luciferase activity of AC16 cells co-transfected with miR-22-3p/scramble controls and wild-type NLRP3 3'UTR compared with mutant NLRP3 3'UTR. "P<0.05 vs. the mutant NLRP3 3'UTR. miR, microRNA; UTR, untranslated; Con, control; WT, wild-type; Mut, mutant.

\section{Discussion}

Since the incidence of massive PE is quite low, no individual physician or hospital can rely on their own experiences to achieve optimal patient management. Despite the application of anticoagulation treatment, the mortality rate of submassive PE doubles in patients suffering from RV dysfunction and preserved systemic arterial pressure (28). Three major factors have been demonstrated to play a role in the clinical manifestation and progression of acute pulmonary embolism: The severity of pre-existing dysfunctions in the cardiopulmonary system, the extent of embolic obstruction in the pulmonary
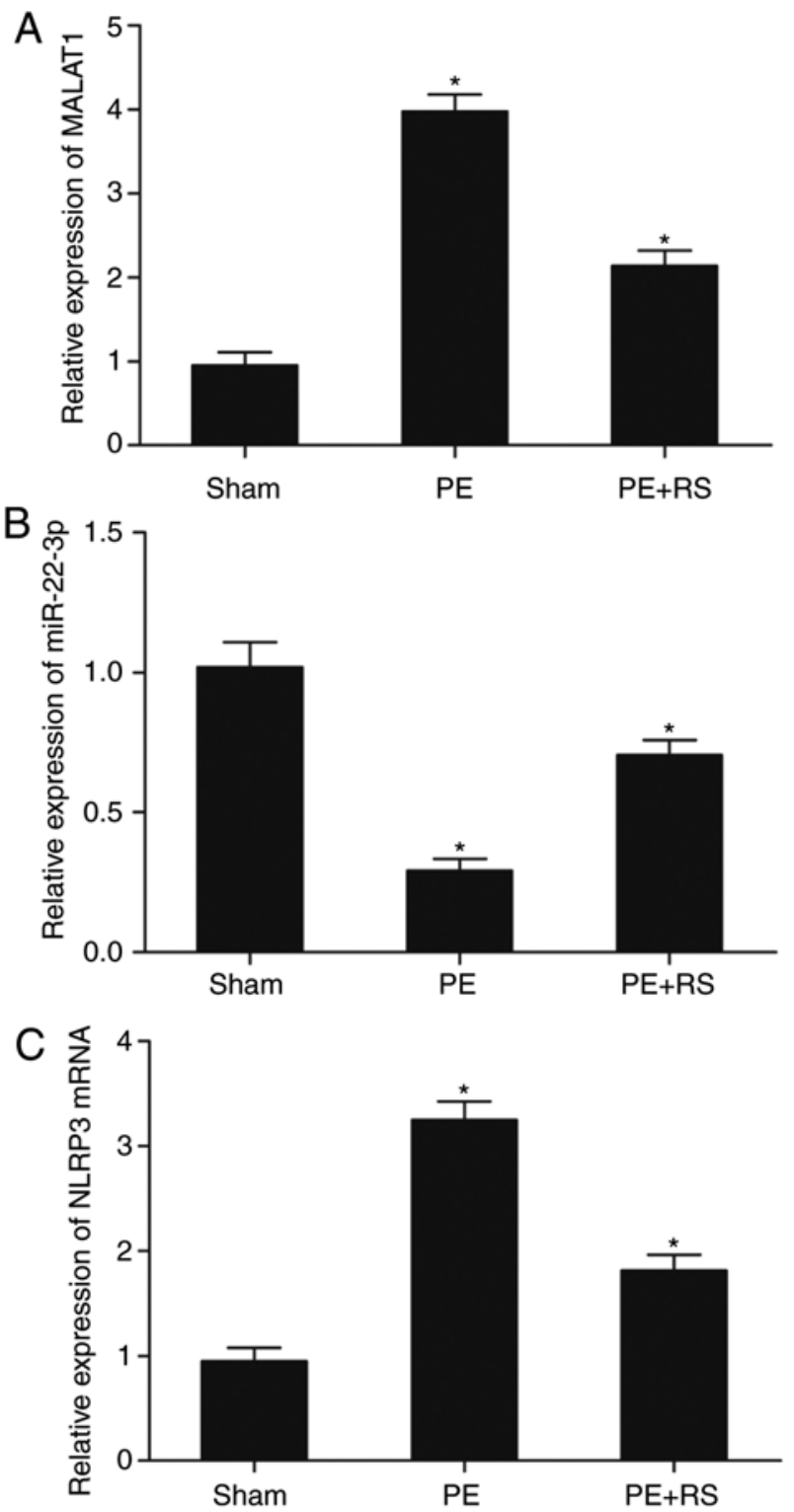

Figure 5. MALAT1, miR-22-3p and NLRP3 are differently expressed in different groups. (A) MALAT1 level in PE associated cardiac injury group, $\mathrm{PE}$ associated cardiac injury plus $\mathrm{RS}$ group and sham group. $\mathrm{P}<0.05$ vs. the sham group. (B) miR-22-3p level in PE associated cardiac injury group, PE associated cardiac injury plus RS group and sham group. ${ }^{*} \mathrm{P}<0.05$ vs. the sham group. (C) NLRP3 level in PE associated cardiac injury group, PE associated cardiac injury plus $\mathrm{RS}$ group and sham group. ${ }^{*} \mathrm{P}<0.05$ vs. the sham group. miR, microRNA; PE, pulmonary embolism; RS, resveratrol.

artery and the presence of thrombosis in peripheral venous vessels, which can potentially induce the recurrence of thromboembolic events $(29,30)$. The interactions between these factors can lead to afterload stress in the RV and the onset of hypertension in the pulmonary artery (29). The presence of above clinical manifestations can be accurately detected using ultrasound imaging (31). It has been demonstrated in previous studies that when the afterload stress in the RV resulted in clinically evident right heart failure (especially when it was also accompanied by symptoms of cardiogenic shock), the prognosis in the patients was poor $(32,33)$.

RS usually exists in the form of cis- and trans- isomers. As the preferred steric form, trans-RS is more stable if protected from light and high $\mathrm{pH}$. The production of trans-RS in plants 
A

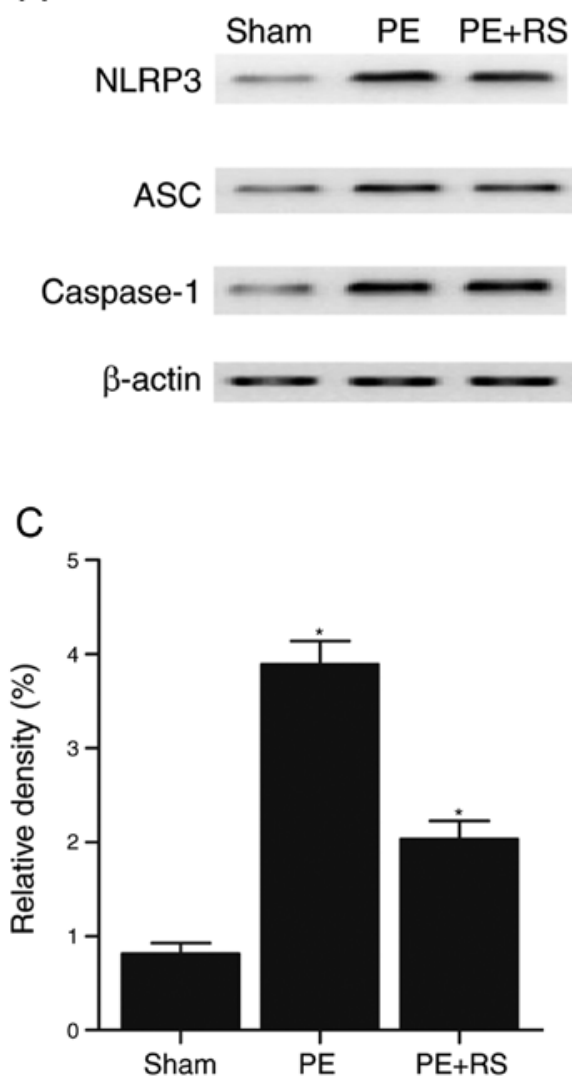

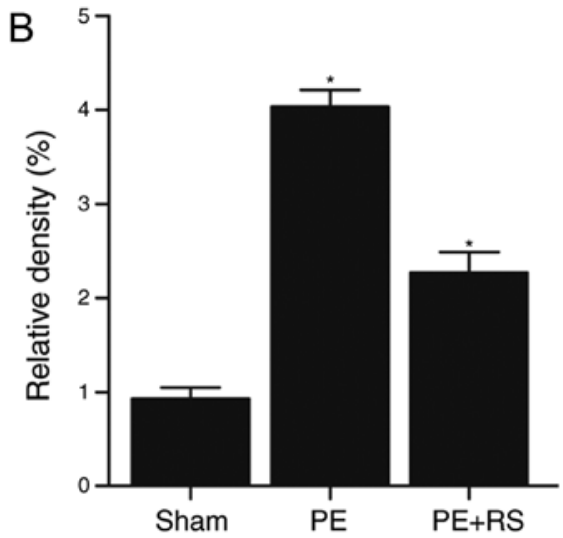

D

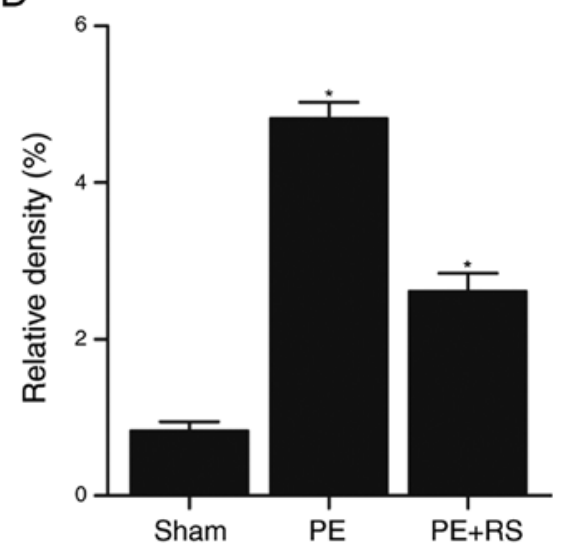

Figure 6. NLRP3, ASC and Caspase-1 are differently expressed in different groups. (A) NLRP3, ASC and Caspase-1 levels in PE associated cardiac injury group, $\mathrm{PE}$ associated cardiac injury plus RS group, and sham group. ${ }^{\mathrm{P}}<0.05$ vs. the sham group. (B) NLRP3 level in PE associated cardiac injury group, $\mathrm{PE}$ associated cardiac injury plus RS group and sham group. ${ }^{*} \mathrm{P}<0.05$ vs. the sham group. (C) ASC level in the PE associated cardiac injury group, PE associated cardiac injury plus RS group and sham group. ${ }^{*} \mathrm{P}<0.05$ vs. the sham group. (D) Caspase-1 level in the PE associated cardiac injury group, $\mathrm{PE}$ associated cardiac injury plus RS group and sham group. "P<0.05 vs. the sham group. PE, pulmonary embolism; RS, resveratrol.
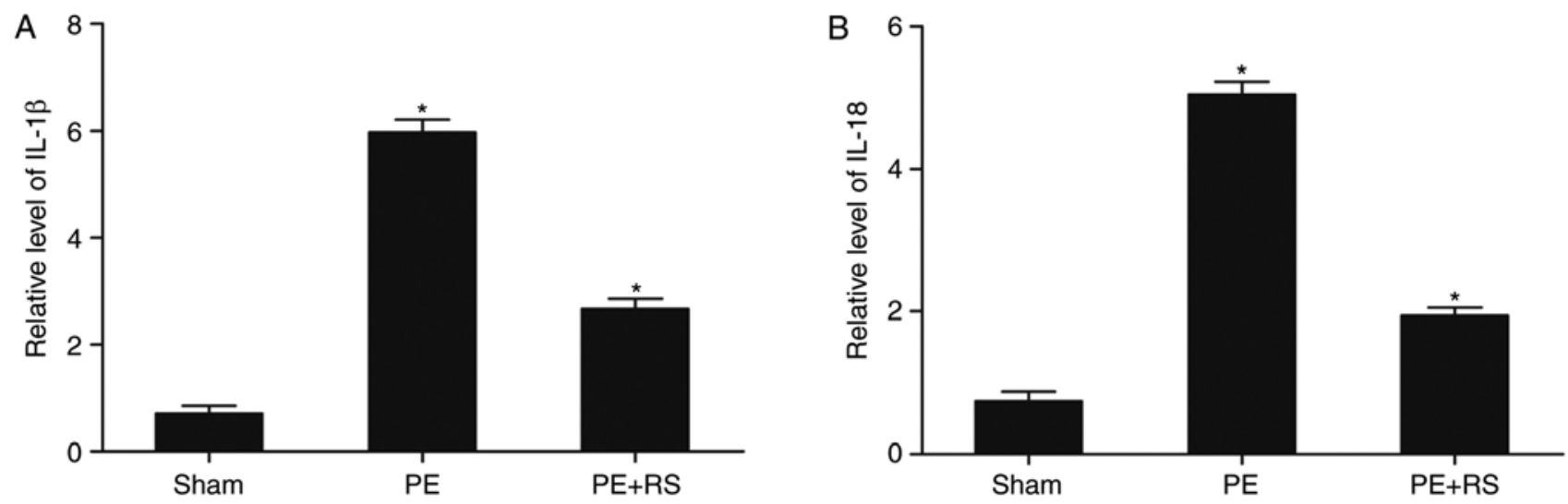

Figure 7. Differential levels of inflammatory factors including IL-1 $\beta$ and IL-18 in various groups. (A) IL-1 $\beta$ level in the PE associated cardiac injury group, $\mathrm{PE}$ associated cardiac injury plus RS group and sham group. ${ }^{*} \mathrm{P}<0.05$ vs. the sham group. (B) IL-18 level in PE associated cardiac injury group, PE associated cardiac injury plus RS group and sham group. ${ }^{*}<0.05$ vs. the sham group. IL, interleukin; PE, pulmonary embolism; RS, resveratrol.

can be triggered by UV radiation, microbial infection, or ozone exposure (34-36). A recent study has demonstrated that RS could improve the ability of spatial learning as well as memory capacity in septic mice. These conclusions were drawn based on the observed inhibition of neuronal apoptosis and the activation of microglia after the administration of RS. In addition, RS was demonstrated to inhibit the NLRP3/IL-1 $\beta$ axis both in vivo and in vitro (37). Several underlying mechanisms are believed to participate in the downregulation of NLRP3 expression and the maturation of IL- $1 \beta$ after the administration of RS. Sirt1 was demonstrated to deacetylate nuclear factor- $\kappa \mathrm{B}$, therefore preventing the nuclear translocation of p65 subunit $(38,39)$. In this study, the underlying mechanism of the therapeutic effect of RS in the treatment of PE associated cardiac injury was explored and it was found that RS upregulated promoter efficiency of MALAT1. Furthermore, 


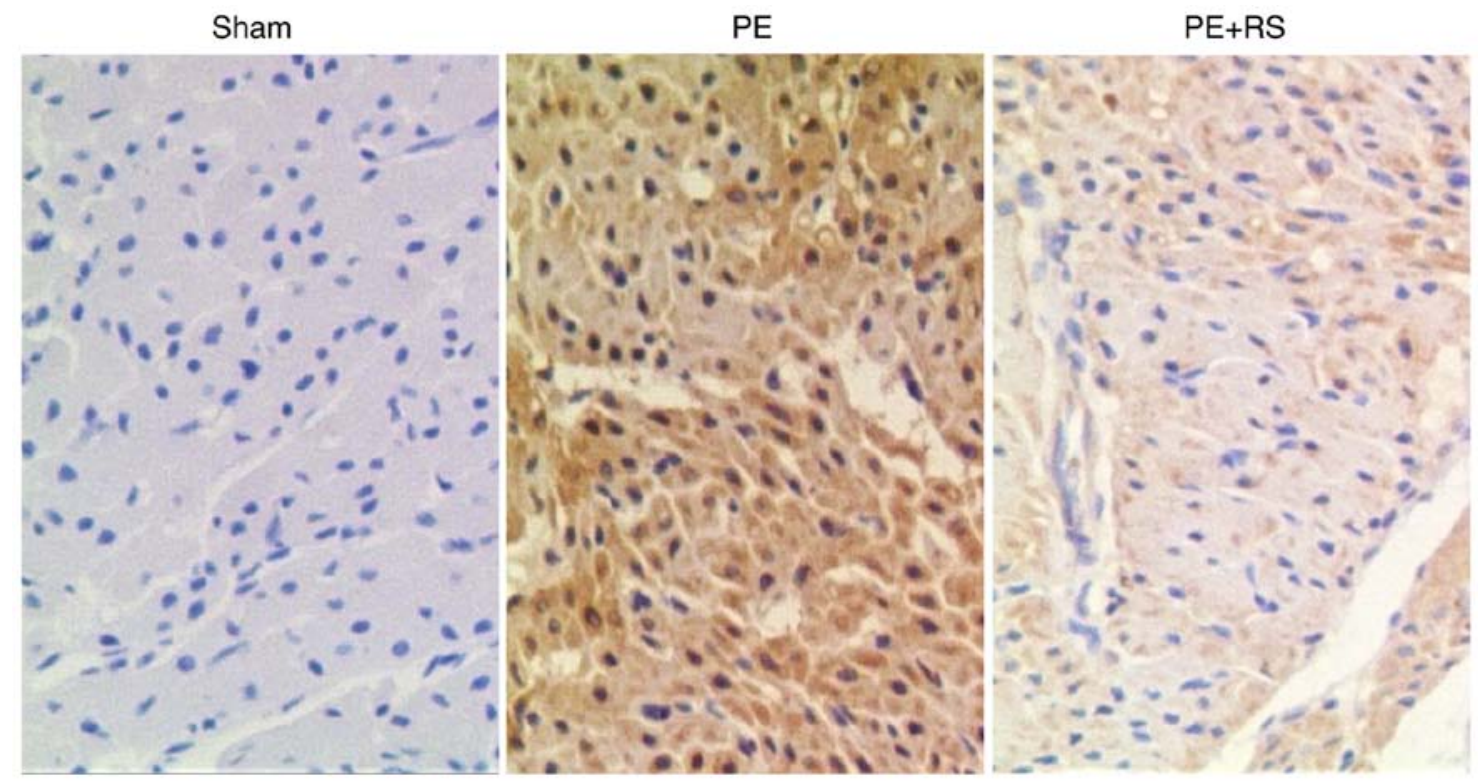

Figure 8. NLRP3 protein in PE associated cardiac injury group, PE associated cardiac injury plus RS group and sham group (magnification, x40). PE, pulmonary embolism; RS, resveratrol.

in-silico analysis was performed to investigate the regulatory relationship between MALAT1 and miR-22-3p, and it was revealed that MALAT1 directly targeted and repressed miR-22-3p expression. In addition, the regulatory relationship between miR-22-3p and NLRP3 was investigated using online publicly available algorithms, and it was found that miR-22-3p inhibited the expression of NLRP3 by binding to complementary sequences in the 3'UTR of NLRP3.

MALAT1, an IncRNA, was first discovered in invasive non-small cell carcinoma and its overexpression has been found in a number of other cancerous tissues, suggesting that MALAT1 plays a role in the invasion and metastasis of cancer (40-43). In the present study, it has been shown that the expression of MALAT1 in 60 colorectal cancer (CRC) tissues was increased compared with in the adjacent normal tissues and the upregulation in MALAT1 expression was correlated with the invasion and metastasis capabilities of CRC. In the screening experiments, the monomers extracted from two Chinese traditional medicines were identified as having a direct effect on the expression of MALAT1. Among these two monomers, the potency of RS was higher. It was demonstrated in a previous study that the inhibition of MALAT1 expression by shRNA significantly reduced the invasion and migration of CRC LoVo and HCT116 cells (43). Similar to the outcome of RS treatment, the downregulation of MALAT1 expression also suppressed the nuclear translocation of $\beta$-catenin, therefore leading to a decreased level of c-Myc and matrix metalloproteinase-7. On the other hand, the upregulation of MALAT1 expression achieved the opposite results (14). Therefore, one key MALAT1 question is to determine the correct conditions of cellular stress and pathological environments so that the presence of MALAT1 in vivo becomes essential (44). A recent study has demonstrated the essential role of MALAT1 in the tight regulation of LPS-induced inflammatory responses, therefore presenting the first piece of evidence supporting the role of MALAT1 in the regulation of inflammation and innate immunity (45). In addition, another study demonstrated that between the livers of wild-type and MALAT1 knockout mice, the only gene with significantly altered expression was serum amyloid A3, an inflammatory mediator (37). In the present study, it was found that MALAT1 and NLRP3 levels were increased, while miR-22-3p level was decreased in the PE associated cardiac injury plus RS group compared with the sham group. In addition, MALAT1 and NLRP3 levels were increased, and miR-22-3p level was decreased in PE associated cardiac injury group compared with the PE associated cardiac injury plus RS group. Furthermore, the present study demonstrated that NLRP3, ASC and Caspase-1 levels in the PE associated cardiac injury plus RS group was increased compared with the sham group, while a greater increase was demonstrated in the PE associated cardiac injury group compared with the PE associated cardiac injury plus RS group.

The activation of inflammasomes, as represented by increased NLRP3 expression, has been shown to be a key process during inflammation (9). Several endogenous signals, such as extracellular adenosine triphosphate, monosodium urate, crystals of calcium phosphates crystals of cholesterols, amyloid- $\beta$, hyaluronan and islet amyloid polypeptide (also known as amylin) can trigger the activation of NLRP3 inflammasomes, whereas exogenous signals such as silica and asbestos can also activate NLRP3 inflammasomes (46-53). The activation of the NLRP3 inflammasomes by these signals of danger can induce the release of IL-1 $\beta$ and subsequent inflammatory responses, therefore leading to the damage in human organs or tissues (9). In this study, it was also revealed that PE associated cardiac injury plus RS group exhibited higher levels of IL-1 $\beta$ and IL-18 compared with the sham group, and the PE associated cardiac injury group displayed higher levels of IL- $1 \beta$ and IL-18 compared with the PE associated cardiac injury plus RS group. Meanwhile, it was demonstrated that NLRP3 was increased in PE associated cardiac injury group compared with the PE associated cardiac injury plus RS group, both of them were increased compared with the sham group. 
In conclusion, it was demonstrated that RS has been shown to prevent the development of cardiac injury induced by $\mathrm{PE}$ via modulating the expression of MALAT1 and further affect miR-22-3p and NLRP3.

\section{Acknowledgements}

Not applicable.

\section{Funding}

No funding was received.

\section{Availability of data and materials}

The datasets used and/or analyzed during the present study are available from the corresponding author on reasonable request.

\section{Authors' contributions}

KY and WL designed the study. WD and YJ collected the literature. KY, WL, WD, YJ, NH, YL, BR and JS collected and analyzed the data, KY and WL wrote the manuscript and the remaining authors read and approved the final manuscript.

\section{Ethics approval and consent to participate}

All experiments were carried out with the agreement of Institutional Animal Care and Use Committee of the First Affiliated Hospital of Chengdu Medical College according to Guide for the Care and Use of Laboratory Animals.

\section{Patient consent for publication}

Not applicable.

\section{Competing interests}

The authors declare they have no competing interests.

\section{References}

1. Goldhaber SZ and Elliott CG: Acute pulmonary embolism: Part I: Epidemiology, pathophysiology, and diagnosis. Circulation 108: 2726-2729, 2003.

2. Goldhaber SZ, Visani L and De Rosa M: Acute pulmonary embolism: Clinical outcomes in the international cooperative pulmonary embolism registry (ICOPER). Lancet 353: 1386-1389, 1999.

3. Kasper W, Konstantinides S, Geibel A, Olschewski M, Heinrich F, Grosser KD, Rauber K, Iversen S, Redecker M and Kienast J: Management strategies and determinants of outcome in acute major pulmonary embolism: Results of a multicenter registry. J Am Coll Cardiol 30: 1165-1171, 1997.

4. Kasper W, Konstantinides S, Geibel A, Tiede N, Krause T and Just H: Prognostic significance of right ventricular afterload stress detected by echocardiography in patients with clinically suspected pulmonary embolism. Heart 77: 346-349, 1997.

5. Konstantinides S, Geibel A, Olschewski M, Heinrich F, Grosser K, Rauber K, Iversen S, Redecker M, Kienast J, Just H and Kasper W: Association between thrombolytic treatment and the prognosis of hemodynamically stable patients with major pulmonary embolism: Results of a multicenter registry. Circulation 96: 882-888, 1997.
6. Kline JA, Hernandez-Nino J, Rose GA, Norton HJ and Camargo CA Jr: Surrogate markers for adverse outcomes in normotensive patients with pulmonary embolism. Crit Care Med 34: 2773-2780, 2006.

7. Stevinson BG, Hernandez-Nino J, Rose G and Kline JA: Echocardiographic and functional cardiopulmonary problems 6 months after first-time pulmonary embolism in previously healthy patients. Eur Heart J 28: 2517-2524, 2007.

8. Watts JA, Gellar MA, Obraztsova M, Kline JA and Zagorski J: Role of inflammation in right ventricular damage and repair following experimental pulmonary embolism in rats. Int J Exp Pathol 89: 389-399, 2008.

9. Takahashi M: NLRP3 inflammasome as a novel player in myocardial infarction. Int Heart J 55: 101-105, 2014.

10. de la Lastra CA and Villegas I: Resveratrol as an antioxidant and pro-oxidant agent: Mechanisms and clinical implications. Biochem Soc Trans 35: 1156-1160, 2007.

11. Olas B and Wachowicz B: Resveratrol, a phenolic antioxidant with effects on blood platelet functions. Platelets 16: 251-260, 2005.

12. Athar M, Back JH, Tang X, Kim KH, Kopelovich L, Bickers DR and Kim AL: Resveratrol: A review of preclinical studies for human cancer prevention. Toxicol Appl Pharmacol 224: 274-283, 2007.

13. Ji Q, Liu X, Fu X, Zhang L, Sui H, Zhou L, Sun J, Cai J, Qin J, Ren J and Li Q: Resveratrol inhibits invasion and metastasis of colorectal cancer cells via MALAT1 mediated Wnt/ $\beta$-catenin signal pathway. PLoS One 8: e78700, 2013.

14. Wang W, Zhu Y, Li S, Chen X, Jiang G, Shen Z, Qiao Y, Wang L, Zheng $P$ and Zhang Y: Long noncoding RNA MALAT1 promotes malignant development of esophageal squamous cell carcinoma by targeting $\beta$-catenin via Ezh2. Oncotarget 7: 25668-25682, 2016.

15. Iyer MK, Niknafs YS, Malik R, Singhal U, Sahu A, Hosono Y, Barrette TR, Prensner JR, Evans JR, Zhao S, et al: The landscape of long noncoding RNAs in the human transcriptome. Nat Genet 47: 199-208, 2015.

16. Derrien T, Johnson R, Bussotti G, Tanzer A, Djebali S, Tilgner H, Guernec G, Martin D, Merkel A, Knowles DG, et al: The GENCODE v7 catalog of human long noncoding RNAs: Analysis of their gene structure, evolution, and expression. Genome Res 22: 1775-1789, 2012.

17. Banfai B, Jia H, Khatun J, Wood E, Risk B, Gundling WE Jr, Kundaje A, Gunawardena HP, Yu Y, Xie L, et al: Long noncoding RNAs are rarely translated in two human cell lines. Genome Res 22: 1646-1657, 2012.

18. Guttman M, Russell P, Ingolia NT, Weissman JS and Lander ES: Ribosome profiling provides evidence that large noncoding RNAs do not encode proteins. Cell 154: 240-251, 2013.

19. Cabili MN, Trapnell C, Goff L, Koziol M, Tazon-Vega B, Regev A and Rinn JL: Integrative annotation of human large intergenic noncoding RNAs reveals global properties and specific subclasses. Genes Dev 25: 1915-1927, 2011.

20. Hezroni H, Koppstein D, Schwartz MG, Avrutin A, Bartel DP and Ulitsky I: Principles of long noncoding RNA evolution derived from direct comparison of transcriptomes in 17 species. Cell Rep 11: 1110-1122, 2015.

21. Quinn JJ, Zhang QC, Georgiev P, Ilik IA, Akhtar A and Chang HY: Rapid evolutionary turnover underlies conserved lncRNA-genome interactions. Genes Dev 30: 191-207, 2016.

22. Ulitsky I, Shkumatava A, Jan CH, Sive H and Bartel DP: Conserved function of lincRNAs in vertebrate embryonic development despite rapid sequence evolution. Cell 147: 1537-1550, 2011.

23. Tang Y, Jin X, Xiang Y, Chen Y, Shen CX, Zhang YC and Li YG: The lncRNA MALAT1 protects the endothelium against ox-LDL-induced dysfunction via upregulating the expression of the miR-22-3p target genes CXCR2 and AKT. FEBS Lett 589: 3189-3196, 2015.

24. Kobayashi M, Usui-Kawanishi F, Karasawa T, Kimura H, Watanabe S, Mise N, Kayama F, Kasahara T, Hasebe N and Takahashi M: The cardiac glycoside ouabain activates NLRP3 inflammasomes and promotes cardiac inflammation and dysfunction. PLoS One 12: e0176676, 2017.

25. Jiang L, Zhang L, Kang K, Fei D, Gong R, Cao Y, Pan S and Zhao M: Resveratrol ameliorates LPS-induced acute lung injury via NLRP3 inflammasome modulation. Biomed Pharmacother 84: 130-138, 2016.

26. Dong W, Yang R, Yang J, Ding J, Wu H and Zhang J: Resveratrol pretreatment protects rat hearts from ischemia/reperfusion injury partly via a NALP3 inflammasome pathway. Int J Clin Exp Pathol 8: 8731-8741, 2015. 
27. Livak KG and Schmittgen TD: Analysis of real-time quantitative PCR data and the 2(-Delta Delta C(T)) method. Methods 25: 402-408, 2001

28. Kucher N, Rossi E, De Rosa M and Goldhaber SZ: Prognostic role of echocardiography among patients with acute pulmonary embolism and a systolic arterial pressure of $90 \mathrm{~mm} \mathrm{Hg}$ or higher. Arch Intern Med 165: 1777-1781, 2005.

29. McIntyre KM and Sasahara AA: Hemodynamic and ventricular responses to pulmonary embolism. Prog Cardiovasc Dis 17: 175-190, 1974.

30. Hull RD, Raskob GE, Coates G, Panju AA and Gill GJ: A new noninvasive management strategy for patients with suspected pulmonary embolism. Arch Intern Med 149: 2549-2555, 1989.

31. Kasper W, Geibel A, Tiede N, Bassenge D, Kauder E, Konstantinides S, Meinertz $\mathrm{T}$ and Just $\mathrm{H}$ : Distinguishing between acute and subacute massive pulmonary embolism by conventional and Doppler echocardiography. Br Heart J 70: 352-356, 1993.

32. Alpert JS, Smith R, Carlson J, Ockene IS, Dexter L and Dalen JE: Mortality in patients treated for pulmonary embolism. JAMA 236: 1477-1480, 1976

33. Hall RJ, Sutton GC and Kerr IH: Long-term prognosis of treated acute massive pulmonary embolism. Br Heart J 39: 1128-1134, 1977.

34. Ignatowicz E and Baer-Dubowska W: Resveratrol, a natural chemopreventive agent against degenerative diseases. Pol J Pharmacol 53: 557-569, 2001.

35. Soleas GJ, Yan J and Goldberg DM: Measurement of trans-resveratrol, (+)-catechin, and quercetin in rat and human blood and urine by gas chromatography with mass selective detection. Methods Enzymol 335: 130-145, 2001.

36. Pervaiz S: Resveratrol: From grapevines to mammalian biology. FASEB J 17: 1975-1985, 2003

37. Sui DM, Xie Q, Yi WJ, Gupta S, Yu XY, Li JB, Wang J, Wang JF and Deng XM: Resveratrol protects against sepsis-associated encephalopathy and inhibits the NLRP3/IL-1 $\beta$ Axis in microglia. Mediators Inflamm 2016: 1045657, 2016.

38. Fu Y, Wang Y, Du L, Xu C, Cao J, Fan T, Liu J, Su X, Fan S, Liu Q and Fan F: Resveratrol inhibits ionising irradiation-induced inflammation in MSCs by activating SIRT1 and limiting NLRP-3 inflammasome activation. Int J Mol Sci 14: 14105-14118, 2013.

39. Garcia JA, Volt H, Venegas C, Doerrier C, Escames G, López LC and Acuña-Castroviejo D: Disruption of the NF- $\mathrm{B} / \mathrm{NLRP} 3$ connection by melatonin requires retinoid-related orphan receptor- $\alpha$ and blocks the septic response in mice. FASEB J 29: 3863-3875, 2015

40. de Selincourt K: A breach of trust? Nurs Times 88: 19, 1992.

41. Lin R, Maeda S, Liu C, Karin M and Edgington TS: A large noncoding RNA is a marker for murine hepatocellular carcinomas and a spectrum of human carcinomas. Oncogene 26: 851-858, 2007
42. Guo F, Li Y,Liu Y, Wang J and Li G: Inhibition of metastasis-associated lung adenocarcinoma transcript 1 in CaSki human cervical cancer cells suppresses cell proliferation and invasion. Acta Biochim Biophys Sin (Shanghai) 42: 224-229, 2010.

43. Xu C, Yang M, Tian J, Wang X and Li Z: MALAT-1: A long non-coding RNA and its important 3 ' end functional motif in colorectal cancer metastasis. Int J Oncol 39: 169-175, 2011.

44. Gutschner T, Hammerle M and Diederichs S: MALAT1-a paradigm for long noncoding RNA function in cancer. J Mol Med (Berl) 91: 791-801, 2013.

45. Zhao G, Su Z, Song D, Mao Y and Mao X: The long noncoding RNA MALAT1 regulates the lipopolysaccharide-induced inflammatory response through its interaction with $\mathrm{NF}-\kappa \mathrm{B}$. FEBS Lett 590: 2884-2895, 2016.

46. Martinon F, Petrilli V, Mayor A, Tardivel A and Tschopp J: Gout-associated uric acid crystals activate the NALP3 inflammasome. Nature 440: 237-241, 2006.

47. Pazar B, Ea HK, Narayan S, Kolly L, Bagnoud N, Chobaz V, Roger T, Liote F, So A and Busso N: Basic calcium phosphate crystals induce monocyte/macrophage IL- $1 \beta$ secretion through the NLRP3 inflammasome in vitro. J Immunol 186: 2495-2502, 2011.

48. Duewell P, Kono H, Rayner KJ, Sirois CM, Vladimer G, Bauernfeind FG, Abela GS, Franchi L, Nunez G, Schnurr M, et al: LRP3 inflammasomes are required for atherogenesis and activated by cholesterol crystals. Nature 464: 1357-1361, 2010.

49. Rajamaki K, Lappalainen J, Oorni K, Valimaki E, Matikainen S, Kovanen PT and Eklund KK: Cholesterol crystals activate the NLRP3 inflammasome in human macrophages: A novel link between cholesterol metabolism and inflammation. PLoS One 5: e11765, 2010

50. Halle A, Hornung V, Petzold GC, Stewart CR, Monks BG, Reinheckel T, Fitzgerald KA, Latz E, Moore KJ and Golenbock DT: The NALP3 inflammasome is involved in the innate immune response to amyloid-beta. Nat Immunol 9: 857-865, 2008.

51. Yamasaki K, Muto J, Taylor KR, Cogen AL, Audish D Bertin J, Grant EP, Coyle AJ, Misaghi A, Hoffman HM and Gallo RL: NLRP3/cryopyrin is necessary for interleukin-lbeta (IL-1beta) release in response to hyaluronan, an endogenous trigger of inflammation in response to injury. J Biol Chem 284: 12762-12771, 2009.

52. Masters SL, Dunne A, Subramanian SL, Hull RL, Tannahill GM, Sharp FA, Becker C, Franchi L, Yoshihara E, Chen Z, et al: Activation of the NLRP3 inflammasome by islet amyloid polypeptide provides a mechanism for enhanced IL-1 $\beta$ in type 2 diabetes. Nat Immunol 11: 897-904, 2010.

53. Dostert C, Pétrilli V, Van Bruggen R, Steele C, Mossman BT and Tschopp J: Innate immune activation through Nalp3 inflammasome sensing of asbestos and silica. Science 320: 674-677, 2008. 